\title{
Tuning the Oxygen Reduction Activity of Pd Shell Nanoparticles with Random Alloy Cores
}

\author{
Liang Zhang and Graeme Henkelman*
}

Department of Chemistry and Biochemistry and the Institute for Computational Engineering and Sciences, The University of Texas at Austin, Austin, Texas 78712-0165, United States

ABSTRACT: Pd-based nanoparticles are promising candidates for non-Pt catalysts of the oxygen reduction reaction (ORR). Trends in ORR activity of $\mathrm{Pd} / \mathrm{Cu}$-alloy-core@Pd-shell nanoparticles are studied by calculating the oxygen binding energy on the Pd surface with different $\mathrm{Cu}$ compositions in the alloy core. Density functional theory calculations show that several properties of the nanoparticle surface, including the average oxygen binding energy, $d$-band center, and the net charge of $\mathrm{Pd}$, are linearly related to the ratio of $\mathrm{Cu}$ in the core, demonstrating the capacity to tune ORR activity. Trends in oxygen binding of other core alloys are also studied and show similar linear trends with core composition, providing a design strategy for new

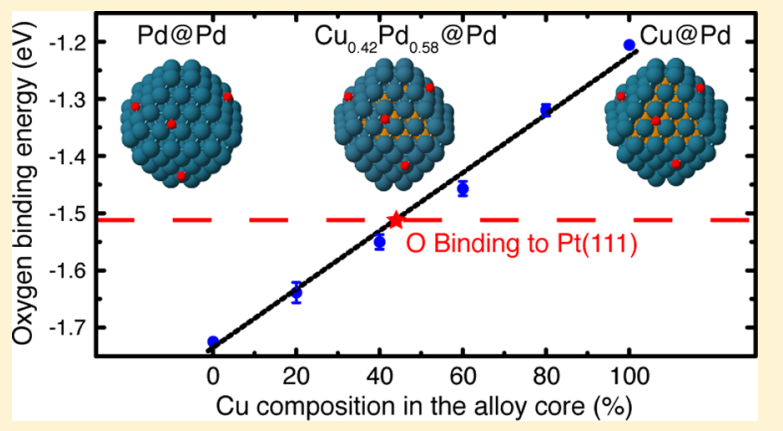
ORR catalysts.

\section{INTRODUCTION}

Fuel cells show promise as a future power source that combines the high chemical energy density of fuels with high-efficiency conversion to electricity and zero or low emissions. The widespread application of current proton exchange membrane fuel cells, however, is impeded by several limitations in the oxygen reduction catalyst at the cathode. As the best catalyst for the oxygen reduction reaction (ORR) so far, Pt-based materials still have some deficiencies, such as slow oxygen reduction kinetics and a high material cost, preventing them from being commercially valuable in large-scale (e.g., automotive) applications. Bimetallic nanoparticles are one of the most attractive candidates for new ORR catalysts that are less expensive and more efficient than Pt.

Core@shell and random alloy are two bimetallic structures that have been studied in the search for Pt alternatives, largely because of their amenability to tune electrical properties by controlling the particle composition. Each structure, however, has its own shortcomings from the standpoint of design and synthesis. For core@shell nanoparticles, variations in composition are discrete in chemical compound space (elements can only be changed by integer atomic numbers), so that the catalytic properties cannot be tuned continuously. ${ }^{1}$ The properties of random alloys can be fine-tuned by varying the composition of the components, ${ }^{2}$ but there are limitations associated with having reactive metals on the particle surface because they can be oxidized and dissolve into solution. Here we show how the advantages of core@shell particles can be combined with those of random alloys in the form of alloycore@shell particles. Noble metal (e.g., Pt and Pd) shells are stable during synthesis and characterization, and the alloy core composition offers the opportunity to tune the electronic structure of the shell.
There have been several experimental studies of the synthesis and ORR activity of alloy-core@shell structures. Wang et al. leached surface $\mathrm{Ni}$ from $\mathrm{Pt} / \mathrm{Ni}$ random alloy nanoparticles to achieve a Pt-bimetallic catalyst with a multilayer Pt-skin surface. $^{3}$ Zhou et al. deposited a $\mathrm{Cu}$ monolayer on a $\mathrm{Pd}_{3} \mathrm{Fe}(111)$ single-crystal alloy via under potential deposition and then replaced the $\mathrm{Cu}$ monolayer with $\mathrm{Pt}$ by galvanic exchange. ${ }^{4}$ Both structures showed high catalytic activity and good durability for the ORR. Recently, Cochell and Manthiram synthesized a series of core-shell nanoparticles with $\mathrm{Pd} / \mathrm{Cu}$ rich alloy cores and Pt-rich shells and studied the effect of varying $\mathrm{Cu}$ content on the ORR activity. ${ }^{5}$ However, theoretical principles for tuning the activity of the shell based upon changing the alloy core composition are not yet wellunderstood.

In this work, trends in the O binding energy on Pd shell nanoparticles with different random alloy cores are investigated to understand how the core composition affects ORR activity. Bligaard et al. reported that $\mathrm{O}$ binding energy is an effective descriptor for ORR activity. ${ }^{6}$ Nørskov et al. showed a volcanoshaped relationship between the ORR rate and the oxygen adsorption energy. ${ }^{7}$ On one side of the volcano peak, $\mathrm{O}$ or $\mathrm{OH}$ strongly bind to the metal so that the reaction rate is limited by the removal of product (hydroxyl). On the other side of the peak, oxygen binds weakly to metals such as $\mathrm{Ag}$ and $\mathrm{Au}$, and the kinetics are limited by high dissociation barriers. According to the volcano plot, the peak in activity is predicted to be at an oxygen binding slightly weaker than on a $\mathrm{Pt}(111)$ surface. In this article, we choose a target oxygen binding energy of the $\mathrm{Pt}(111)$ surface, $-1.51 \mathrm{eV}$, with respect to gas-phase $\mathrm{O}_{2}$, as

Received: June 1, 2012

Revised: September 13, 2012

Published: September 24, 2012 
Table 1. Oxygen Binding Energy of Pd-shell NP140

\begin{tabular}{|c|c|c|c|c|c|c|c|c|c|}
\hline \multicolumn{9}{|c|}{ Pd-shell 140 nanoparticles } & \multirow[b]{3}{*}{$\operatorname{Pt}(111)$ slab } \\
\hline \multirow[b]{2}{*}{ core metal } & \multicolumn{3}{|c|}{ group I } & \multicolumn{5}{|c|}{ group II } & \\
\hline & $\mathrm{Au}$ & $\mathrm{Ag}$ & $\mathrm{Pd}$ & Ir & $\mathrm{Rh}$ & $\mathrm{Cu}$ & $\mathrm{Ru}$ & Mo & \\
\hline$B E_{\mathrm{O}}(\mathrm{eV})$ & -1.81 & -1.75 & -1.72 & -1.46 & -1.41 & -1.21 & -1.12 & -0.94 & -1.51 \\
\hline
\end{tabular}

calculated on a four-layer $3 \times 3$ slab model. Although oxygen binding on bulk $\mathrm{Pt}(111)$ is not necessarily optimal for ORR, it gives a close enough estimate of the location of the volcano peak to understand trends in nanoparticle activity.

\section{COMPUTATIONAL MODELS AND METHODS}

In this study, the binding energy of oxygen to the Pd shell was calculated with DFT, as implemented in the Vienna ab initio simulation package., ${ }^{8,9}$ Core electrons were described using the projector-augmented wave method. ${ }^{10,11}$ Kohn-Sham singleelectron wave functions were expanded in a plane-wave basis with a kinetic energy cutoff of $275 \mathrm{eV}$ to describe the valence electrons. The generalized gradient approximation (GGA) using the Perdew-Wang 91 functional $^{12}$ was chosen to evaluate the exchange-correlation energy. Spin-polarization was tested and was used when necessary. All atoms in the nanoparticle were allowed to relax; geometries were considered optimized when the force on each atom was $<0.005 \mathrm{eV} / \AA$. Convergence was checked by increasing the energy cutoff to $400 \mathrm{eV}$, and the oxygen binding energy on a Pd 140-atom nanoparticle was found to change by only $1 \mathrm{meV}(<0.1 \%)$.

Alloy-core@Pd-shell nanoparticles were modeled as facecentered cubic (FCC) crystallites in the shape of truncated octahedron containing 140 atoms (denoted as NP140) with 44 core atoms and 96 shell atoms. We denote the Pd-shell nanoparticles with random alloy cores as $\mathrm{X}_{x} \mathrm{Y}_{1-x} @ \mathrm{Pd}$, where $\mathrm{X}$ and $\mathrm{Y}$ are the two metals constituting the core, and $x$ is the fraction of metal X. A cubic box of side length $26 \AA$ was used to contain the particle with a vacuum gap of at least $11 \AA$ in all directions to avoid interactions from periodic images. $А$ sampling of the Brillouin zone was used for the isolated particles. For each configuration, an oxygen atom was adsorbed on each of the eight central FCC hollows of the (111) facets. The $\mathrm{O}$ binding energy $E b$ was calculated by averaging over these eight sites,

$$
E b=\frac{1}{8}\left(E_{\mathrm{NP}+8 \mathrm{O}}-E_{\mathrm{NP}}-4 E_{\mathrm{O}_{2}}\right)
$$

where $E_{\mathrm{NP}+8 \mathrm{O}}$ is the energy of the particle with eight oxygen atoms adsorbed, $E_{\mathrm{NP}}$ is the energy of the bare particle, and the energy of the $\mathrm{O}_{2}$ molecule is chosen as the reference for oxygen. Ten different random-alloy configurations were generated to calculate the average $\mathrm{O}$ binding energy for each core composition ( 80 binding sites).

To help understand trends in O binding, we used Bader's atoms-in-molecules approach, in which the total charge density is partitioned by zero-flux surfaces. ${ }^{13}$ A grid-based version of this algorithm was used to quantify changes in atomic charges in the Pd shell due to alloying in the core. ${ }^{14}$ The local density of state (DOS) was also calculated to determine the average $d$ band center and width of the Pd shell.

\section{RESULTS AND DISCUSSION}

Oxygen Binding Energy. Table 1 lists the $\mathrm{O}$ binding energy of various kinds of Pd-shell NP140 with a single core element. Compared with a Pt(111) slab, these Pd-shell NP140s can be classified into two groups. Group I includes the strong $\mathrm{O}$ binding structures: Au@Pd, Ag@Pd, and Pd@Pd, whereas Group II contains structures with weaker $\mathrm{O}$ binding: $\mathrm{Ir} @ \mathrm{Pd}$, Rh@Pd, Cu@Pd, Ru@Pd, and Mo@Pd. The more noble the core metal, the more reactive the corresponding Pd-shell nanoparticles. For instance, $\mathrm{Au}$ itself is known to have the weakest $\mathrm{O}$ binding among all of the transitional metals; however, Au@Pd binds O most strongly of the Pd-shell particles.

We first take $\mathrm{Cu}_{x} \mathrm{Pd}_{1-x} @ \mathrm{Pd} \mathrm{NP} 140$ as a case study. The two extremes of $\mathrm{Cu}_{x} \mathrm{Pd}_{1-x} @ \mathrm{Pd}(x=0$ and 1$)$ are pure $\mathrm{Pd}$ and $\mathrm{Cu} @$ $\mathrm{Pd}$ particles, which are on opposite sides of the peak in the volcano plot. Both are expected to have lower activity as compared with $\mathrm{Pt}(111)$; the $\mathrm{Pd}$ particle binds $\mathrm{O}$ too strongly, whereas $\mathrm{Cu} @ \mathrm{Pd}$ binds $\mathrm{O}$ too weakly. When the composition of the core is varied, however, we have an opportunity to find an optimal alloy-core@shell structure that has the target $\mathrm{O}$ binding.

Figure 1 shows the average binding energy of atomic oxygen on $\mathrm{Cu}_{x} \mathrm{Pd}_{1-x} @ \mathrm{Pd} \mathrm{NP} 140$. A near-linear relationship between

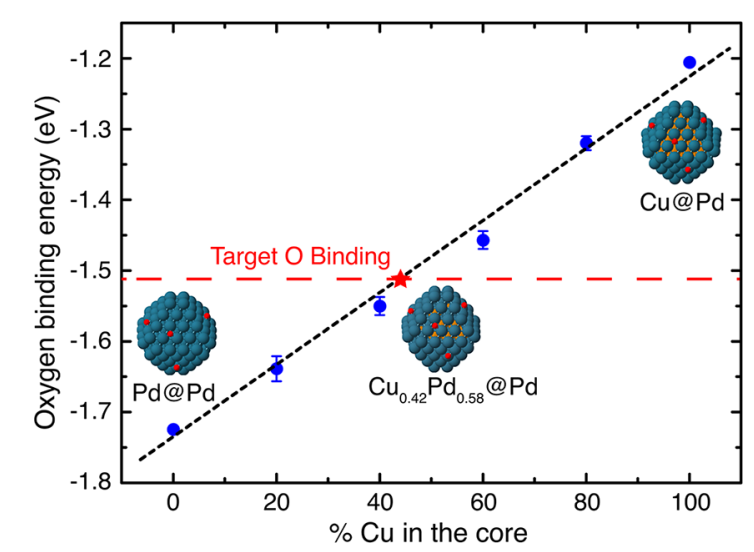

Figure 1. Average binding energy of oxygen to the $\mathrm{Pd}(111)$ facets of $\mathrm{Pd} / \mathrm{Cu}$ alloy-core@Pd shell nanoparticles. The inset figures show the $\mathrm{Pd}$ (blue) shelled nanoparticles with varying $\mathrm{Cu}$ (orange) composition in the core as well as the oxygen (red) binding sites.

the average atomic $\mathrm{O}$ binding energy and $\mathrm{Cu}$ ratio in the alloy core is shown in the Figure. The red dashed line marks the $\mathrm{O}$ binding energy on $\mathrm{Pt}(111)$, which is the target $\mathrm{O}$ binding. Each $\mathrm{O}$ binding energy data point is averaged over 10 different configurations with the specified core composition. The standard deviation of the $\mathrm{O}$ binding distribution is indicated by the error bars. Using the $\mathrm{O}$ binding energy as a descriptor for ORR activity and $\mathrm{O}$ binding on $\mathrm{Pt}(111)$ as the target, we are able to predict a volcano-shaped relationship between ORR activity and the $\mathrm{Cu}$ ratio in the alloy core with its peak at $42 \%$ $\mathrm{Cu}$ in the alloy core. Because there are 44 atoms in the core of a 140-atom particle, we predict that $\mathrm{Cu}_{18} \mathrm{Pd}_{26} @ \mathrm{Pd}$ should give the best catalytic activity for the ORR. 
Compared with a previous study of $\mathrm{Pd} / \mathrm{Cu}$ random alloy nanoparticles, $\mathrm{Cu}_{x} \mathrm{Pd}_{1-x} @ \mathrm{Pd} \mathrm{NP} 140$ have two distinct features in the trend of $\mathrm{O}$ binding energy as a function of composition. ${ }^{2}$ First, unlike the quadratic relationship between $\mathrm{O}$ binding energy and $\mathrm{Cu}$ composition calculated for $\mathrm{Pd} / \mathrm{Cu}$ random alloys, the relationship for $\mathrm{Cu}_{x} \mathrm{Pd}_{1-x} @ \mathrm{Pd} \mathrm{NP} 140$ is linear. Second, the distribution of $\mathrm{O}$ binding energies on $\mathrm{Cu}_{x} \mathrm{Pd}_{1-x} @$ $\mathrm{Pd}$ is much narrower than that for $\mathrm{Pd} / \mathrm{Cu}$ random alloys. These two distinctions arise from the homogeneity of the shell composition. In $\mathrm{Pd} / \mathrm{Cu}$ random alloys, the average $\mathrm{O}$ binding energy varies linearly with the binding energy to the component metals, and the significance of these contributions to the average varies linearly with composition. The product of these two factors leads to the quadratic $\mathrm{O}$ binding trend with alloy composition. In the $\mathrm{Pd}$ shell particles, there is no variation in the species to which $\mathrm{O}$ binds - it is always $\mathrm{Pd}-$ and so the $\mathrm{O}$ binding trend is linear in composition. Similarly, the distribution of $\mathrm{O}$ binding energy is large in the $\mathrm{Pd} / \mathrm{Cu}$ random alloy particles where O binds to FCC sites on the (111) facets, which have four distinct compositions $\left(\mathrm{Pd}_{3}, \mathrm{Pd}_{2} \mathrm{Cu}_{1}, \mathrm{Pd}_{1} \mathrm{Cu}_{2}\right.$, and $\mathrm{Cu}_{3}$ ). In $\mathrm{Cu}_{x} \mathrm{Pd}_{1-x} @ \mathrm{Pd} \mathrm{NP} 140$, the FCC sites always consist of three $\mathrm{Pd}$ atoms, which reduces the variance of the $\mathrm{O}$ binding energy distribution.

$\boldsymbol{d}$-Band Model. The $d$-band model proposed by Hammer and Nørskov has been widely used to understand trends in chemisorption of adsorbates on the transition-metal surface. ${ }^{15-17}$ In this model, the interaction between the metal surface and the adsorbate molecule is described as a function of the $d$-band of the metal. When a shift in $d$-band center of metal surface is the dominant factor determining chemisorption strength, a differential change in chemisorption energy is linearly correlated to the shift in the position of $d$-band center. $^{17,18}$ In the case of $\mathrm{Pd}-\mathrm{O}$ interaction, this linear relationship can be expressed as

$$
\delta E_{\mathrm{Pd}-\mathrm{O}} \simeq-4 f_{d} \frac{V^{2}}{\left|\varepsilon_{d}-\varepsilon_{\mathrm{O}}\right|^{2}} \delta \varepsilon_{d}
$$

where $f_{d}$ is the local filling of the Pd $d$-states, $\varepsilon_{\mathrm{O}}$ is the center of the oxygen $2 \mathrm{p}$ states, and $V$ is the coupling matrix element between the oxygen $2 \mathrm{p}$ orbitals and $\mathrm{Pd} d$-states. When comparing similar binding geometries, changes in $f_{d}$ and $V$ can be small as compared with the $d$-band center shift so that in a small range of $\varepsilon_{d}$ the slope $\delta E_{\mathrm{Pd}-\mathrm{O}} / \delta \varepsilon_{d}$ can be approximated as a constant.

Figure 2 shows the linear relationship between the average $d$ band center of $\mathrm{Pd}$ atoms on (111) facets and the $\mathrm{Cu}$ ratio in the nanoparticle core. Increasing the $\mathrm{Cu}$ ratio in the alloy core lowers the $d$-band center of the shell. As the $d$-band center is lowered from the Fermi-level $\left(E_{\mathrm{F}}\right)$, there is less overlap between the $d$-states of the surface Pd atoms and the $2 \mathrm{p}$ states of the adsorbed $\mathrm{O}$, resulting in weaker $\mathrm{O}$ binding. Therefore, the linear $\mathrm{O}$ binding trend calculated with DFT can be explained by the linear shift in the $d$-band center with $\mathrm{Cu}$ concentration in the core.

To understand better the relationship between the $d$-band center and core composition, it is helpful to determine the factors that affect the $d$-band center. In the discussion by Tang et al. on core-shell nanoparticles, ${ }^{1}$ it was found that changes of $d$-band filling and $d$-bandwidth are two important factors that shift the $d$-band center. Change of the $d$-band filling is mainly due to charge redistribution between the Pd surface atoms and the alloy core; change of the $d$-bandwidth is attributed to bond-

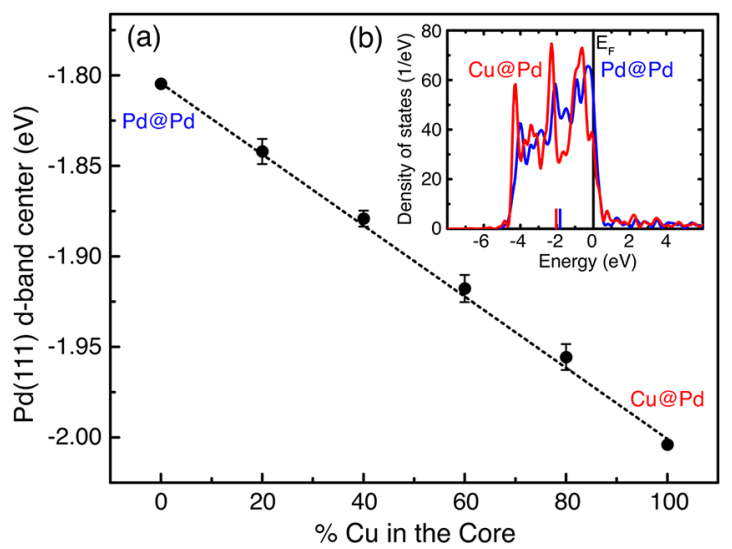

Figure 2. (a) Average $d$-band center of Pd atoms on the (111) facets is lowered by the addition of $\mathrm{Cu}$ in the core, which in turn weakens the $\mathrm{O}$ binding energy. (b) $d$-band density of states for $\mathrm{Pd}$ (111) atoms in the two extremes: Pd@Pd (blue) and Cu@Pd (red). The small vertical marks above the $x$-axis indicate the center of the $d$-bands. The $\mathrm{Cu}$ core lowers the $d$-band of the Pd shell as compared with the Pd core.

length variation in the surface (the strain effect) and electronic overlap with subsurface alloy atoms (the ligand effect). Using the approach of Tang et al., we were able to determine by how much the $d$-band center shift is caused by charge redistribution and how much it is caused by the widening of the surface $d$ band.

Figure 3 shows the amount of charge transferred to Pd atoms on (111) facets with respect to the pure Pd@Pd nanoparticle.

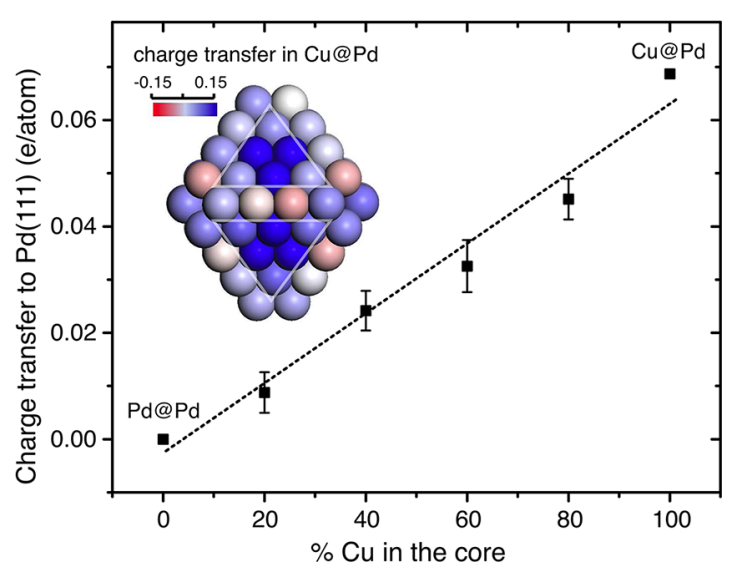

Figure 3. Average net charge on Pd (111) facet atoms (indicated by triangles in the inset figure) in alloy core nanoparticles as compared with monometallic $\mathrm{Pd}$ particles as a function of the $\mathrm{Cu}$ ratio in the core. The inset shows the Bader charge redistribution on the Pd shell atoms due to the change of core metal from $\mathrm{Pd}$ to $\mathrm{Cu}$.

The charge was calculated as an integration of valence charge within Bader volumes surrounding each Pd atom. As shown in Figure 3, the net charge on the surface $\mathrm{Pd}$ atoms is proportional to the ratio of $\mathrm{Cu}$ in the alloy core. Because $\mathrm{Cu}$ has a higher Fermi energy than $\mathrm{Pd}{ }^{19}$ charge flows from $\mathrm{Cu}$ to $\mathrm{Pd}$, when $\mathrm{Cu}$ is alloyed in the core of the nanoparticles. Since the number of $\mathrm{Pd}$ shell atoms is fixed at 96 , more $\mathrm{Cu}$ in the alloy core results in more net charge distributed on the $\mathrm{Pd}$ atoms. The transferred charge from $\mathrm{Cu}$ to $\mathrm{Pd}$ then increases the filling of the $\mathrm{Pd} d$-band and lowers the $d$-band center with respect to the Fermi level. This lowering of $d$-band can be estimated by dividing the DOS at the Fermi level into the amount of charge 
transferred. The DOS of the Pd (111) surface atoms at the Fermi level is 52 states/eV for Pd@Pd and 35 states/eV for $\mathrm{Cu} @ P d$. Considering that the total number of Pd (111) facet atoms is 48 , the average Pd DOS at the Fermi level is 43 states/ $\mathrm{eV}$ or 0.89 states/eV/atom. As shown in Figure 3, comparing Pd@Pd to Cu@Pd, 0.07 e/atom of charge flows from Cu in the subsurface to $\mathrm{Pd}$ in the shell. Using the above values, the effect of charge transfer is estimated to lower the $d$-band center by $0.08 \mathrm{eV}$.

Figure 4 shows a linear increase in the $d$-bandwidth with the ratio of $\mathrm{Cu}$ in the alloy core. Because $\mathrm{Cu}$ has a smaller lattice

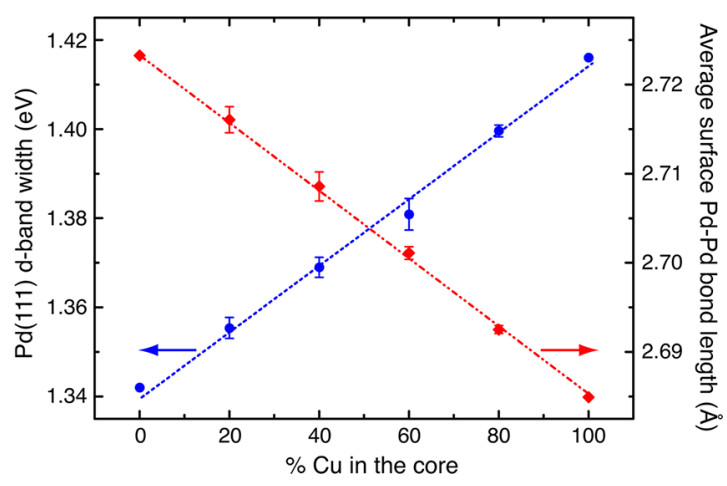

Figure 4. Trends of average $d$-bandwidth (blue) and average $\mathrm{Pd}-\mathrm{Pd}$ bond length (red) of $\mathrm{Pd}(111)$ facet atoms as a function of the ratio of $\mathrm{Cu}$ in the alloy core.

constant than $\mathrm{Pd}$, alloying $\mathrm{Cu}$ into the core shrinks the size of the nanoparticle, causing a shorter Pd-Pd bond length in the $\mathrm{Pd}$ shell. The average Pd-Pd bond length between Pd atoms on the (111) facets decreases linearly with the ratio of $\mathrm{Cu}$ in the alloy core, from 2.725 to $2.685 \AA$. The compression of the PdPd bond enhances the overlap between states and widens the $d$ band. The effect of $d$-band widening on the shift of the $d$-band center can be estimated in a rectangular model of the $d$-band. ${ }^{20}$ The correlation between these two $d$-band character parameters are given by

$$
\delta \varepsilon_{d}=\sqrt{12}\left(0.5-f_{d}\right) \delta w
$$

where $\delta w$ is the change of $d$-bandwidth, $\delta \varepsilon_{d}$ is the corresponding shift of $d$-band center, and $f_{d}$ is the filling of $\mathrm{Pd} d$-band. As shown in Figure 4, the $d$-band width increases by $0.08 \mathrm{eV}$ with the replacement of $\mathrm{Pd}$ for $\mathrm{Cu}$ in the core. The average filling of the Pd NP140 $d$-band is 0.91 . Using eq 3 , the lowering of the $d$-band center due to band widening is estimated to be $0.11 \mathrm{eV}$.

From the above analysis, replacing $\mathrm{Cu}$ in the core of a $\mathrm{Pd}$ particle linearly increases the Pd shell $d$-band filling and the Pd $d$-bandwidth. These two effects are estimated to lower the $d$ band center of $\mathrm{Pd}$ (111) facet atoms by 0.08 and $0.11 \mathrm{eV}$, respectively. The total estimated $d$-band shift from these two factors is in excellent agreement with what is calculated explicitly $(0.2 \mathrm{eV})$. Our results are consistent with the finding of Tang et al. on core-shell nanoparticles; charge transfer can be an important factor for tuning the $d$-band structure of small nanoparticles.

Oxygen Binding to Other $\mathrm{M}_{x} \mathrm{Pd}_{1-x} @ \mathrm{Pd} \mathrm{NP} 140$. Obinding trends of other $\mathrm{M}_{x} \mathrm{Pd}_{1-x} @ \mathrm{Pd} \mathrm{NP} 140$ were studied to examine the universality of the linear relation between $O$ binding energy trend and core component ratio. Because Pd@ $\mathrm{Pd}$ binds $\mathrm{O}$ stronger than the target $\mathrm{Pt}(111)$ slab, we selected metals M belonging to Group II in Table 1 for which M-core@ $\mathrm{Pd}$-shell particles bind $\mathrm{O}$ weaker than the target to tune the $\mathrm{O}$ binding of the Pd-shell particles toward the target value. The choice of such metals to mix with Pd in the core can be Ir, Rh, $\mathrm{Cu}, \mathrm{Ru}$, or Mo. Figure 5 shows that each $\mathrm{O}$ binding energy

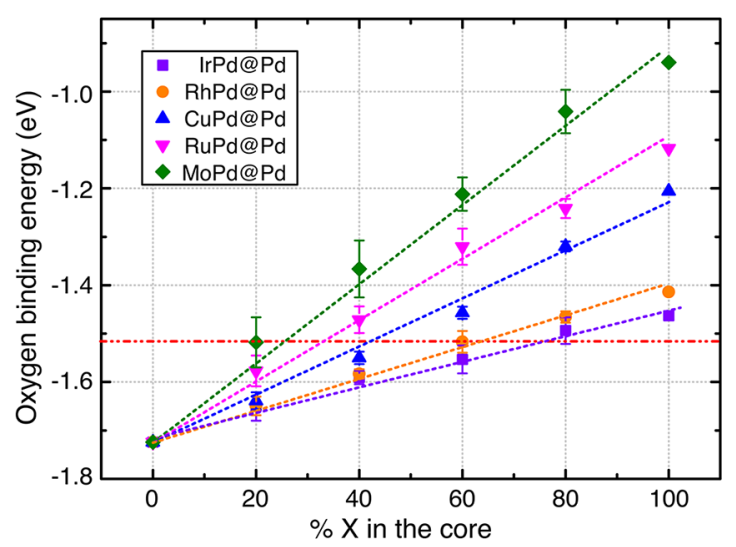

Figure 5. $\mathrm{O}$ binding to various $\mathrm{M}_{x} \mathrm{Pd}_{1-x} @ \mathrm{Pd} \mathrm{NP140}(\mathrm{M}=\mathrm{Ir}, \mathrm{Rh}, \mathrm{Cu}$, $\mathrm{Ru}$ and $\mathrm{Mo}$ ).

trend is linear with the ratio of the alloying metal $\mathrm{M}$ in the core. Furthermore, in the case study of $\mathrm{Cu}_{x} \mathrm{Pd}_{1-x} @ \mathrm{Pd} \mathrm{NP} 140$, the electronic structure (e.g., $d$-band center) of the Pd shell was also observed to vary linearly with the alloy-core composition. Therefore, we expect that the linear binding trend is a general picture of the adsorbate binding to alloy-core@shell nanoparticles, which can be applied to design new catalysts for various catalytic reactions on nanoparticles.

With the validation of this linear $\mathrm{O}$ binding trend, the target $\mathrm{O}$ binding of a Pd-shell particle can be achieved by alloying a metal $\mathrm{X}$ from the second group $(\mathrm{X}=\mathrm{Ir}, \mathrm{Rh}, \mathrm{Cu}, \mathrm{Ru}, \mathrm{Mo})$ with metal $\mathrm{Y}$ from the first group $(\mathrm{Y}=\mathrm{Au}, \mathrm{Ag}, \mathrm{Pd})$ in core. The optimal ratio $\mathrm{x}^{*}$ for the $\mathrm{X}_{x} \mathrm{Y}_{1-x} @ \mathrm{Pd}$ nanoparticle is

$$
x^{*}=\frac{E b_{\mathrm{Y} @ \mathrm{Pd}}-E b_{\mathrm{Pt}(111)}}{E b_{\mathrm{Y} @ \mathrm{Pd}}-E b_{\mathrm{X} @ \mathrm{Pd}}}
$$

where $E b_{\mathrm{Pt}(111)}$ is the $\mathrm{O}$ binding energy on a $\mathrm{Pt}(111)$ slab, which we chose as the target, and $E b_{\mathrm{X} @ \mathrm{Pd}}$ and $E b_{\mathrm{Y} @ \mathrm{Pd}}$ are the $\mathrm{O}$ binding of core-shell NP140 from Table 1. Using eq 4, a simple prediction of the optimal ratio $x^{*}$ for all possible core component combinations is given in Table 2.

Stability of Pd-Shell Nanoparticles. Stability is another important property for nanoparticle catalysts. The thermodynamic stability of a core-shell structure is largely dependent on the relative surface energies and adsorbate binding energies of the component elements. ${ }^{21,22}$ Previous studies have used these energies as a measure of near-surface alloy stability. Ruban et al.

Table 2. Optimal Ratio $x^{*}$ of Metal X Alloyed with Metal Y in the Core of a Pd-Shelled Particle

\begin{tabular}{lccc} 
& \multicolumn{3}{c}{ metal Y } \\
\cline { 2 - 4 } metal X & $\mathrm{Au}$ & $\mathrm{Ag}$ & $\mathrm{Pd}$ \\
$\mathrm{Ir}$ & 0.86 & 0.83 & 0.81 \\
$\mathrm{Rh}$ & 0.75 & 0.71 & 0.68 \\
$\mathrm{Cu}$ & 0.49 & 0.44 & 0.41 \\
$\mathrm{Ru}$ & 0.43 & 0.38 & 0.35 \\
$\mathrm{Mo}$ & 0.34 & 0.29 & 0.27
\end{tabular}


constructed a comprehensive database of surface segregation energies to study the satiability of transition-metal solute/host overlayers in vacuum. ${ }^{23}$ Greeley and Mavrikakis also considered adsorbate binding in their investigations of the stability of nearsurface alloys in the presence of $\mathrm{CO}$ and $\mathrm{H}^{24}$ Here we follow the same strategy and calculate core/shell segregation energies, $E_{\text {seg }}$ of the (111) facet atoms of the Pd-shell particles. Specifically, we calculated $E_{\text {seg }}$ as the energy required to swap a Pd atom on (111) facet with a neighboring core atom, as illustrated in Figure 6. The segregation energy with bound $\mathrm{O}$ was also computed to model stability in the environment of the ORR.

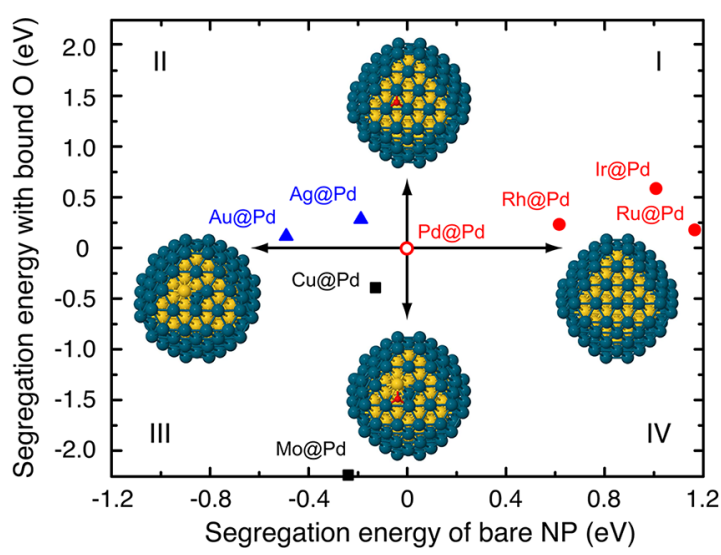

Figure 6. Stability of Pd-shell particles with various core elements. The insets indicate the stable structures along each axis direction.

The nanoparticles in quadrant I of Figure 6 (Ir@Pd, Rh@Pd, and $\mathrm{Ru} @ \mathrm{Pd}$ ) have positive segregation energies and are therefore expected to be stable in both vacuum and an $\mathrm{O}$ rich environment. In quadrant II, the core elements $\mathrm{Au}$ and $\mathrm{Ag}$ would prefer the surface in vacuum, but the stronger binding of O to Pd will stabilize the Au@Pd and Ag@Pd structures in the presence of oxygen. This calculation is consistent with experiments done in the Crooks group in which dendrimerencapsulated Au@Pd nanoparticles are found to be stable in water and under ORR where oxygen species are expected to be bound on the surface. ${ }^{25}$ In quadrant III, the Cu@Pd and Mo@ Pd particles are not expected to be thermodynamically stable.

On the basis of these calculations, many of the alloy-core particles with optimal O binding energies, including PdX@Pd ( $\mathrm{X}=\mathrm{Ir}, \mathrm{Rh}$ and $\mathrm{Ru}$ ), are expected to be thermodynamically stable. Particles with Ag and Au cores, including AgX@Pd and $\mathrm{AuX} @ \mathrm{Pd}(\mathrm{X}=\mathrm{Ir}, \mathrm{Rh}$ and $\mathrm{Ru})$, are also expected to be stable in the O-rich environment of the ORR. The particles with Mo and $\mathrm{Cu}$ present in the cores are likely the least stable; these elements are more stable on the surface of the particle and may leach into solution when oxidized. Such particles should not be ruled out; however, $\mathrm{Cu}$ was found to be kinetically stable in the core of Pd particles and more active in ORR experiments than pure Pd particles. ${ }^{5}$

\section{CONCLUSIONS}

A linear trend was found for the $\mathrm{O}$ binding energy to $\mathrm{Cu}_{x} \mathrm{Pd}_{1-x} @ \mathrm{Pd} \mathrm{NP140s}$ as a function of $\mathrm{Cu}$ ratio in the alloy core. To approach the high ORR activity of a $\mathrm{Pt}(111)$ slab, the $O$ binding energy on this surface was chosen as our target. The ORR activity of $\mathrm{Cu}_{x} \mathrm{Pd}_{1-x} @ \mathrm{Pd}$ is then predicted to reach a peak when the alloy core is composed of $42 \% \mathrm{Cu}$. Further study of the $d$-band model shows that the mixing of $\mathrm{Cu}$ in the core linearly increases the $d$-band filling and width, which in turn leads to the linear $\mathrm{O}$ binding trend. These two contributions are roughly equivalent in magnitude, and their net effect on the $d$-band center is in agreement with what is observed from direct DFT calculation. The same linear $\mathrm{O}$ binding trend is found in other X/Y-alloy-core@Pd-shell particles, providing a set of alloy-core compositions with a Pd-shell that archive the target O binding of $\mathrm{Pt}(111)$. This class of alloy-core@shell nanoparticles combine the robustness of core@shell particles with the tunability of alloy particles. Our simple model of $\mathrm{O}$ binding to these nanoparticles provides a guideline for designing efficient non-Pt ORR catalyst. The linear trends are expected to hold for other adsorbates binding to this class of alloy-core@ shell particles so that the tuning principle with alloy composition will be applicable to other catalytic reactions.

\section{AUTHOR INFORMATION}

\section{Corresponding Author}

*E-mail: henkelman@mail.utexas.edu.

\section{Notes}

The authors declare no competing financial interest.

\section{ACKNOWLEDGMENTS}

This work was supported by the U.S. Department of Energy, Office of Basic Energy Sciences (grant DE-FG02-09ER16090). Computing time was provided by the National Energy Research Scientific Computing Center and the Texas Advanced Computing Center at the University of Texas at Austin.

\section{REFERENCES}

(1) Tang, W.; Henkelman, G. J. Chem. Phys. 2009, 130, 194504.

(2) Tang, W.; Zhang, L.; Henkelman, G. J. Phys. Chem. Lett. 2011, 2, $1328-1331$

(3) Wang, C.; et al. J. Am. Chem. Soc. 2011, 133, 14396-14403.

(4) Zhou, W.-P.; Yang, X.; Vukmirovic, M. B.; Koel, B. E.; Jiao, J.; Peng, G.; Mavrikakis, M.; Adzic, R. R. J. Am. Chem. Soc. 2009, 131, 12755-12762.

(5) Cochell, T.; Manthiram, A. Langmuir 2012, 28, 1579-1587.

(6) Bligaard, T.; Nørskov, J. K.; Dahl, S.; Matthiesen, J.; Christensen, C. H.; Sehested, J. J. Catal. 2004, 224, 206-217.

(7) Nørskov, J. K.; Rossmeisl, J.; Logadottir, A.; Lindqvist, L.; Kitchin, J. R.; Bligaard, T.; Jónsson, H. J. Phys. Chem. B 2004, 108, 17886-17892.

(8) Kresse, G. Phys. Rev. B 2000, 62, 8295-8305.

(9) Kresse, G.; Hafner, J. Surf. Sci. 2000, 459, 287-302.

(10) Blöchl, P. E. Phys. Rev. B 1994, 50, 17953.

(11) Kresse, G.; Joubert, D. Phys. Rev. B 1999, 59, 1758.

(12) Perdew, J. P.; Wang, Y. Phys. Rev. B 1992, 45, 13244-13249.

(13) Bader, R. F. W. Atoms in Molecules: A Quantum Theory; Oxford University Press: New York, 1990.

(14) Tang, W.; Sanville, E.; Henkelman, G. J. Phys.: Condens. Matter 2009, 21, 84204.

(15) Hammer, B.; Nørskov, J. K. Adv. Catal. 2000, 45, 71-129.

(16) Hammer, B.; Nørskov, J. K. Surf. Sci. 1995, 343, 211-220.

(17) Ruban, A.; Hammer, B.; Stoltze, P.; Skriver, H. L.; Nørskov, J. K. J. Mol. Catal. A: Chem. 1997, 115, 421-429.

(18) Xu, Y.; Ruban, A. V.; Mavrikakis, M. J. Am. Chem. Soc. 2004, 126, 4717-4725.

(19) Sigalas, M.; Papaconstantopoulos, D. A.; Bacalis, N. C. Phys. Rev. B 1992, 45, 5777.

(20) Kitchin, J.; Nørskov, J. K.; Barteau, M. A.; Chen, J. G. J. Chem. Phys. 2004, 120, 10240-10246.

(21) Ferrando, R.; Jellinek, J.; Johnston, R. L. Chem. Rev. 2008, 108, 845-910. 
(22) Wang, L.-L.; Johnson, D. D. J. Am. Chem. Soc. 2009, 131, 14023-14029.

(23) Ruban, A. V.; Skriver, H. L.; Nørskov, J. K. Phys. Rev. B 1999, 59, 15990-16000.

(24) Greeley, J.; Mavrikakis, M. Catal. Today 2006, 111, 52-58.

(25) Weir, M. G.; Knecht, M. R.; Frenkel, A. I.; Crooks, R. M. Langmuir 2010, 26, 1137-46. 